\title{
Nanoindentation of patterned metal lines on a Si substrate
}

\author{
Yoonjoon Choi, Subra Suresh * \\ Department of Materials Science and Engineering, Massachusetts Institute of Technology, Cambridge, MA 02139, USA
}

Received 10 June 2002; received in revised form 21 August 2002; accepted 22 August 2002

\begin{abstract}
A systematic experimental study of nanoindentation on unidirectionally patterned aluminum lines on a Si substrate has been conducted. The results show that the observed increase in indentation compliance with decreasing line width cannot be rationalized on the basis of continuum considerations. A simple mechanistic model based on discrete dislocations is suggested.
\end{abstract}

(C) 2002 Acta Materialia Inc. Published by Elsevier Science Ltd. All rights reserved.

Keywords: Nanoindentation; Mechanical properties; Plastic deformation; Thin films; Patterned lines

\section{Introduction}

The inelastic mechanical properties of smallvolume structures depend on both the characteristic structural length scale and overall physical dimensions of structures. This dependence is increasingly apparent when the feature size is reduced to the micrometer scale. For example, a 1 $\mu \mathrm{m}$ thick, continuous metallic film on a substrate exhibits a yield stress of several hundred $\mathrm{MPa}$, whereas the bulk yield stress of the same material is 3-10 times smaller [1]. Such size dependence of mechanical response has primarily been studied thus far in the context of continuous thin films on substrates. These trends cannot be directly extended to assess the mechanical response of patterned features as, for example, when a film is

\footnotetext{
${ }^{*}$ Corresponding author. Tel.: +1-6172533320; fax: +16172530868.

E-mail address: ssuresh@mit.edu (S. Suresh).
}

patterned into stripes or lines or islands. Patterned features constitute structures which have small lengths in more than one direction, and their mechanical response under different confining conditions remains largely unexplored due to the complexity of stress states within the structure and the deficiency of appropriate experimental techniques to characterize local deformation.

Nanoindentation has been utilized extensively to probe mechanical properties of thin films [2-4]. As this technique can generate large strain (or stress) within the relatively small volume of materials, nanoindentation can be extended to other small-volume structures as well, such as patterned lines and islands on substrates. A particularly appealing attribute of nanoindentation is that, at least in principle, it offers a means to probe the elastic and plastic deformation characteristics as well as residual stresses in small features that are patterned onto substrates under constraints, which are representative of real applications including micro-electro-mechanical systems (MEMS) and 
microelectronic devices and packages. However, the interpretation of material response during indentation remains a complex issue [5,6]. In this paper, we present instrumented nanoindentation results of patterned aluminum lines on a silicon substrate, to explore the effects of two length scales (line width and thickness) on the indentation loaddisplacement $(P-h)$ response.

\section{Experimental procedures}

A polycrystalline thin film of $\mathrm{Al}-1.5 \mathrm{wt} . \% \mathrm{Si}, 1$ $\mu \mathrm{m}$ in thickness, was sputter deposited on a 525 $\mu \mathrm{m}$ thick Si substrate. Subsequently, the film was patterned into sets of parallel lines with different widths using standard photolithography and dry etching techniques. The nominal line width was varied from 1.5 to $5.0 \mu \mathrm{m}$ with an incremental increase in width of $0.5 \mu \mathrm{m}$, for a total number of eight line width sets. The length of lines was 500 $\mu \mathrm{m}$. Each set of lines contained seven lines of the same width, with an inter-line spacing equal to line width (Fig. 1(a)). A square pad of $500 \mu \mathrm{m} \times$ $500 \mu \mathrm{m}$ was patterned next to the sets of lines to represent a continuous thin film. Each of the sets was separated by $20 \mu \mathrm{m}$. All structures were constructed on the same wafer by a single set of deposition and etching processes such that all characteristics of film and lines are expected to be the same, except for the in-plane dimensional length scale (i.e. line width). The thin film microstructure was examined by transmission electron microscopy (TEM). The average grain size in the film was $\sim 0.6 \mu \mathrm{m}$, and the grains exhibited typical columnar structure with a (1 111$)$ texture.

Nanoindentation experiments were carried out with a three-sided diamond Berkovich indenter with a rounded tip mounted in NanoTest 600 indenter (MicroMaterials, Ltd., Wrentham, UK). Load-controlled, depth-sensing nanoindentations covering over a complete loading and unloading sequence were conducted on the thin film pad and lines of different widths, to maximum depths of 100 and $200 \mathrm{~nm}$. A dwell period of $10 \mathrm{~s}$ was imposed at the maximum depth, prior to unloading. Fig. 1(b) shows a series of indents at the center of the top surface of a $3 \mu \mathrm{m}$ wide line. All indenta-
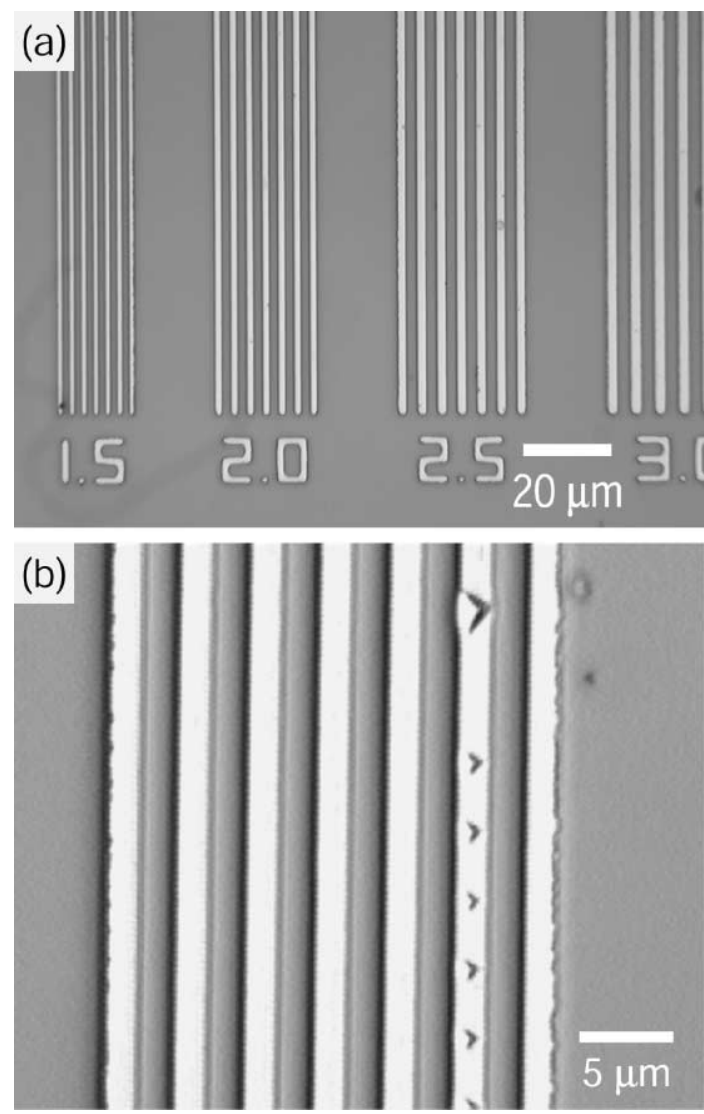

Fig. 1. Micrographs of (a) the patterned lines of polycrystalline $\mathrm{Al}$ on a Si substrate, and (b) a series of indents at the center of the top surface of $3 \mu \mathrm{m}$ wide Al line. The maximum depth of indentation was $500 \mathrm{~nm}$ to demonstrate large indents. All indentations were oriented in the same way with respect to the line direction.

tions were oriented identically with respect to the line direction. The positioning of the indenter in the middle of the line is an experimental challenge, which has been successfully overcome in the present experiments.

\section{Results and discussion}

Typical curves of load $P$ as a function of penetration depth $h$ from the nanoindentation response of the film and lines of three different line widths are presented in Fig. 2. The initial portion 


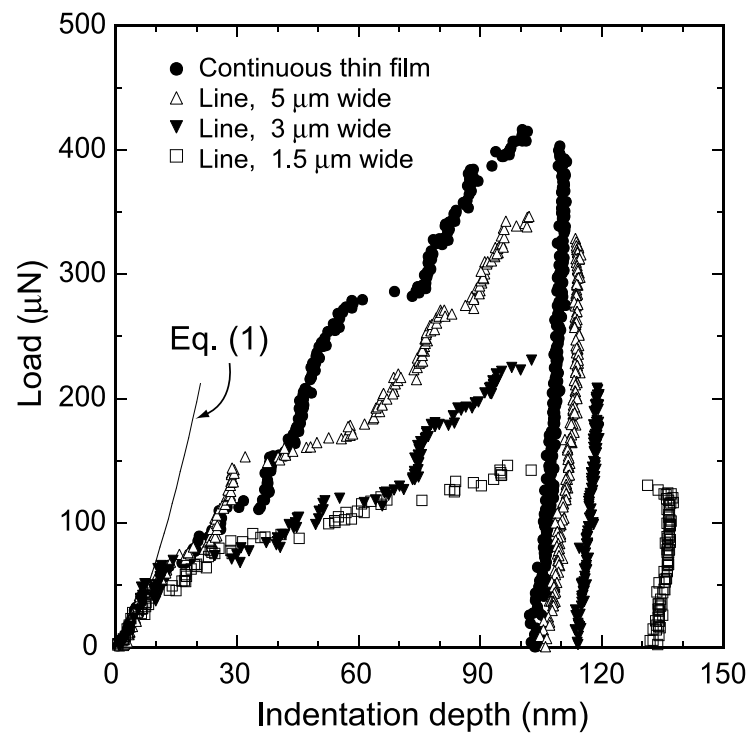

Fig. 2. Load-displacement $(P-h)$ responses of polycrystalline $\mathrm{Al}$ thin film and lines of $1.5,3.0$, and $5.0 \mu \mathrm{m}$ line widths. The solid line denotes the elastic response on aluminum of a spherical diamond indenter with the tip radius $R=500 \mathrm{~nm}$.

of the $P-h$ curves follows the elastic response for a spherical indentation [6]:

$P=\frac{4}{3} E^{*} h^{3 / 2} R^{1 / 2}, \quad \frac{1}{E^{*}}=\frac{1-v_{1}^{2}}{E_{1}}+\frac{1-v_{2}^{2}}{E_{2}}$,

where $R$ is the radius of indenter tip, $E^{*}$ is the reduced elastic modulus of the specimen and indenter, and $E_{1}$ and $E_{2}$ are the elastic moduli of the specimen and indenter, respectively, and $v_{1}$ and $v_{2}$ are the Poisson's ratio of the specimen and indenter, respectively. The value of $E^{*}$ is $74 \mathrm{GPa}$ for polycrystalline $\mathrm{Al}$ indented by a diamond indenter. The initial elastic $P-h$ response matched well with Eq. (1) when the spherical tip radius is assumed to be $\sim 500 \mathrm{~nm}$. The initial deviation from the elastic response, which occurs over a load range of 45-65 $\mu \mathrm{N}$, is approximately independent of line width $w$. The indentation compliance, which can be represented by the curvature of loading response, is also unaffected by line width prior to the initial deviation. The corresponding maximum shear stresses underneath the indenter tip are 1.8-2.0 GPa at the point of deviation, which compares well with the theoretical shear strength of $\mathrm{Al}(\sim 2.5 \mathrm{GPa})$, simi- lar to the trends documented for continuous films of $\mathrm{Al}$ and $\mathrm{Cu}[4,6]$.

Each of these $P-h$ curves exhibits discrete changes (jumps) in the load versus displacement response after the initial deviation from the elastic response as observed in continuous thin films. The propensity for discrete deformation, commonly associated with defect nucleation, is reduced with decreasing line width (for a fixed line thickness). Unlike the elastic response, the $P-h$ response subsequent to this initial deviation is a function of line width $w$. The curvature of the loading response decreases with decreasing $w$, indicating a greater indentation compliance for smaller line width. ${ }^{1}$ The discrete discontinuities in the $P-h$ response are not as clear as those observed in the indentation of single crystalline continuous films $[4,6]$. This contrast may arise from the polycrystalline nature of the present material system, as the combination of grain boundaries and grain orientations can alter the motion of dislocations (or plastic flow) during deformation. The penetration depth during a holding period of $10 \mathrm{~s}$ at maximum load also increases with decreasing line width. However, the unloading slope in Fig. 2 is the same for all line widths.

The penetration depth $h$ at a particular level of imposed load can be considered as a measure of the indentation compliance of the material structures to quantify the loading portion of the $P-h$ response. Fig. 3 shows the statistical variation of indentation depths of the continuous thin film and lines at a fixed indentation load of $200 \mu \mathrm{N}$. The mean penetration depths $h_{\text {mean }}$ are indicated in Fig. 3 . It is evident that narrower lines deform more easily under the same indentation load, resulting in a greater indentation depth. The indentation depth of a $5 \mu \mathrm{m}$ wide line is almost equal to that of the

\footnotetext{
${ }^{1}$ Experimental studies (e.g., Refs. [3,6]) have shown that the yield strength of continuous thin films generally increases significantly with decreasing film thickness. In the present experiments, the width of the patterned lines has been systematically changed for fixed line thickness. Consequently, the trends associated with the softening of the indentation response with increasing line width cannot be directly compared with the effects of film thickness of yield response in continuous thin films.
} 


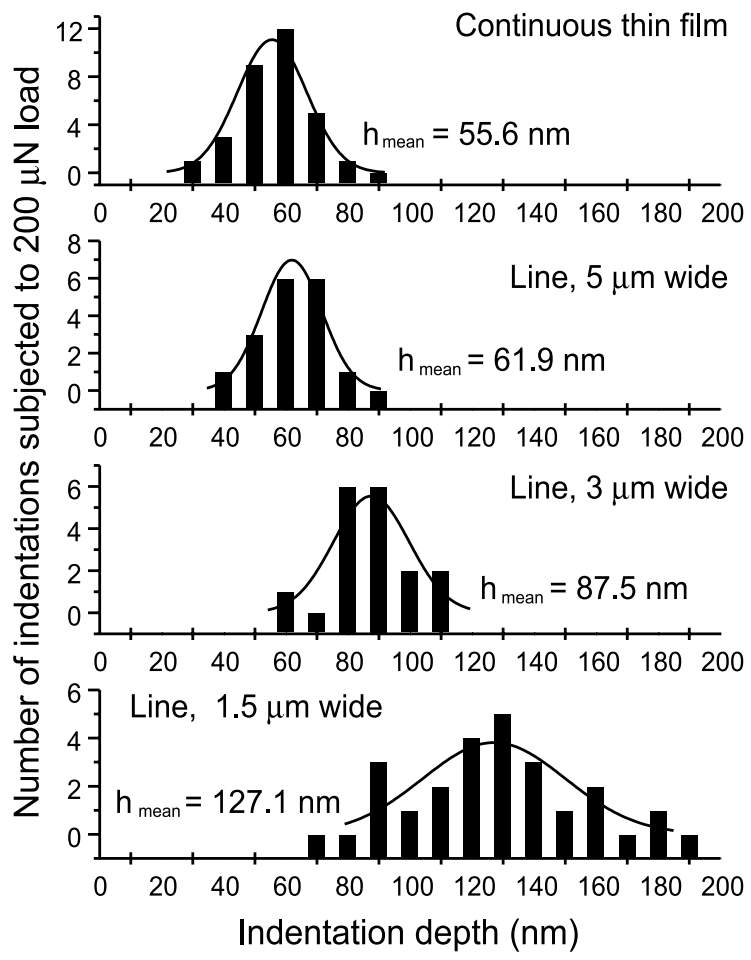

Fig. 3. Statistical distribution of indentation depth at $200 \mu \mathrm{N}$ indentation load for polycrystalline $\mathrm{Al}$ thin film and lines with $1.5,3.0$, and $5.0 \mu \mathrm{m}$ line widths. The mean penetration depths of indentation $h_{\text {mean }}$ are indicated near the normal distribution curves, which are denoted by the solid lines.

continuous film, and the average indentation depth increases as line width decreases. The variation of indentation depths in a given set of experiments for a particular line width also increases as line width decreases. Indentation penetration depth as a function of line width for 100 and 200 $\mu \mathrm{N}$ indentation loads is summarized in Fig. 4.

One possible explanation for the observed increase in compliance with decreasing line width is the interaction of the elasto-plastic boundary beneath the indenter with the free side-wall surfaces of the patterned lines. It has been shown $[5,7]$ that a force balance of Johnson's elasto-plastic indentation model can be used to estimate the plastic zone size, $c$, as a function of the applied load and tensile yield strength, $\sigma_{\mathrm{ys}}$ of the material as:

$c=\sqrt{\frac{3 P}{2 \pi \sigma_{\mathrm{ys}}}}$.

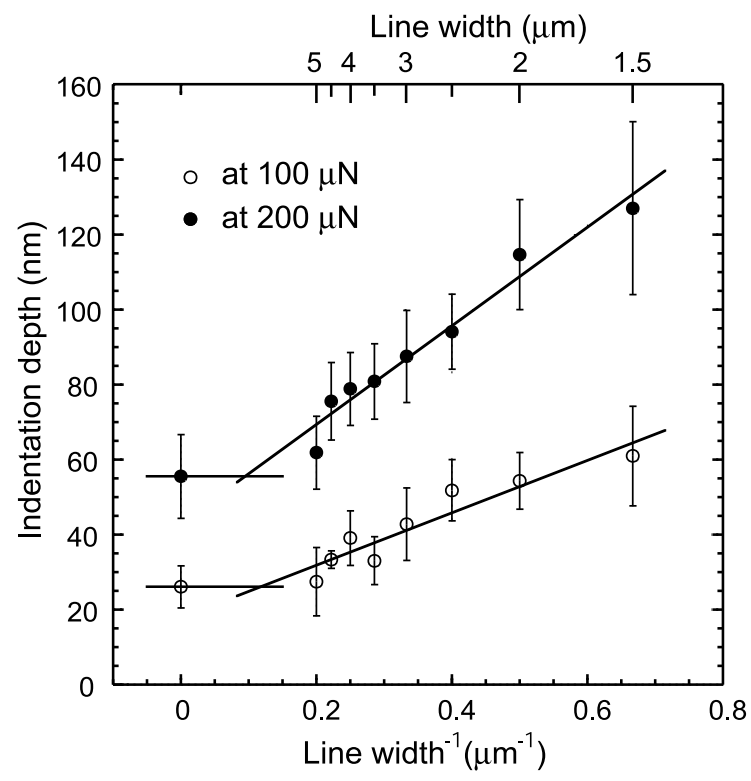

Fig. 4. Indentation depth as a function of line width for 100 and $200 \mu \mathrm{N}$ indentation loads. The error bars indicate the standard deviation of at least 15 indentations.

From Eq. (2), the plastic zone size is $0.56 \mu \mathrm{m}$ for an indentation load of $200 \mu \mathrm{N}$ when $\sigma_{\mathrm{ys}}$ is assumed to be $300 \mathrm{MPa}$ [1]. Since the stress outside the plastic zone vanishes quickly with increasing distance from the indenter tip $\left(\sigma \sim r^{-3}\right)$ [8], the zone size is expected to be similar for the film and lines. Finite element modeling (FEM) [9] of a sharp conical indentation on the continuous film, as well as on 2 and $3 \mu \mathrm{m}$ wide lines of the same $1 \mu \mathrm{m}$ thickness on a $\mathrm{Si}$ substrate, indicates that the plastic zone sizes are $0.57-0.58 \mu \mathrm{m}$ for the film and lines, which agree well with the analytical prediction. The corresponding $P-h$ curves in Fig. 5 show that the response of these cases are essentially indistinguishable up to a load of $400 \mu \mathrm{N}$.

The initial stage of nanoindentation is often explained by the 'punch-out' of dislocation loops around the indenter tip [4,5]. When the indenter depth increases, the dislocation density increases by the nucleation of new dislocations and/or the expansion of existing dislocations. The indenter tip displacement should be accommodated by the plastic flow beneath the indenter tip which is directly related to the number and position of dislocations. The interference between the stress field 

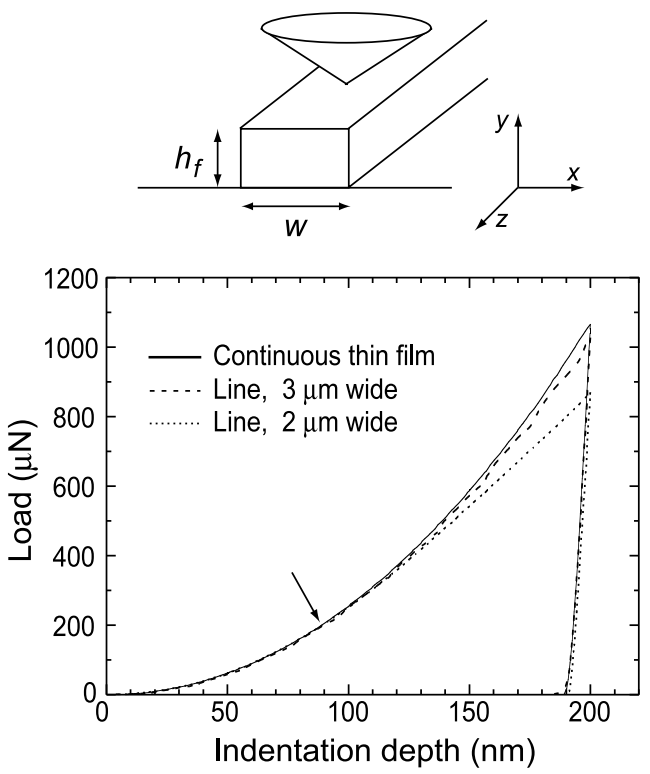

Fig. 5. Simulated load-displacement $(P-h)$ responses of Al thin film and lines using the general purpose finite element package ABAQUS [9]. The indenter was a sharp conical rigid indenter with the included half angle of $70.3^{\circ}$, and $\sigma_{\mathrm{ys}}$ of film and lines is assumed to be $300 \mathrm{MPa}$. The arrow denotes the load and depth level in which we are interested. A schematic of the line geometry along with the appropriate nomenclature is provided at the top of the figure.

of dislocations and the free surfaces of the lines could be considered if the 'punched-out' dislocations can extend their equilibrium position beyond the continuum elasto-plastic boundary. This supposition appears to be a feasible mechanism, in light of the recent result of nanoindentations on $\mathrm{Al}$ and $\mathrm{Cu}$ films $[4,6]$. In these experiments, the $P-h$ curves are affected by film thickness even at shallow depths of indentation $\left(h \leqslant 0.1 h_{\mathrm{f}}\right)$ which are generally considered small enough to avoid substrate effects. Since the $P-h$ response of single crystalline Al films of two distinct thickness $(0.6$ and $1.0 \mu \mathrm{m})$ are distinguishable at a load of $30 \mu \mathrm{N}$ $(c \approx 0.22 \mu \mathrm{m})$ [4], dislocations would be expected to extend as much as five times beyond the continuum plastic zone. Using this line of reasoning, the dislocation extension in our experiments can be as much as $2.8 \mu \mathrm{m}$ at $200 \mu \mathrm{N}$ load. This distance agrees well with pattern line half-width (since $c$ is the radius of plastic zone) at which the $P-h$ re- sponse of lines begins to deviate from that of the film.

Considering that those dislocations are generated near the indenter tip and that they glide along the slip plane to the side surfaces, the effect of dislocation glide on the indenter tip penetration depth in the lines can be rationalized in the following manner. For simplicity, consider a patterned line with dislocations parallel to the line direction. Assuming that the dislocations move in the cross-sectional plane of the line, (which has height of $h_{\mathrm{f}}$ and width $w$, as shown in the schematic in Fig. 5), the additional plastic shear strain of the cross-section becomes $\gamma=\left(2 x b_{\mathrm{x}} / w h\right)$ [10], where $b_{x}$ is the projected magnitude of the Burgers vector along $x$ direction and $x$ is the dislocation glide distance parallel to the line surface. When there are $N$ dislocations that move over the average glide distance $\lambda$, the plastic strain around the indenter tip is given by $2 \lambda N b_{\mathrm{x}} / w h$. This additional strain due to dislocation movement along the line width direction is negligible in the case of continuous films for which $w=\infty$. For the case of patterned lines, however, the material displacement around the indenter tip accompanying this strain becomes $2 \lambda N b_{\mathrm{x}} / w$ for a fixed film thickness of $h_{\mathrm{f}}$. As a first order approximation, the total indentation depth in the line is assumed to be a linear sum of the displacement due to dislocation generation along the indentation direction $\left(h_{\text {film }} \sim N b_{\mathrm{y}}\right.$, where $b_{\mathrm{y}}$ is the projected magnitude of the Burgers vector along the indentation direction), and the additional displacement of the tip due solely to the geometrical effect of the dislocation movement parallel to the line surface. Thus the total indentation depth in the lines becomes:

$h_{\text {line }}=h_{\text {film }}+h_{\text {geo }}=h_{\text {film }}+\alpha \frac{2 \lambda N b_{\mathrm{x}}}{w}$,

where $\alpha$ is a geometrical constant that includes the relative direction of dislocation motion with respect to the indentation axis. The inverse linear effect of line width on the indentation depth in Fig. 4 is captured by Eq. (3). Assuming that the number of generated dislocations $N$ is the same for both films and lines, the average glide distance of dislocation $\lambda$ can be evaluated from the experimental slope of Eq. (3) in Fig. 4. The number $N$ 
can be estimated by the indentation depth deviation from the elastic curve. Taking $\alpha \sim 1$ and $b_{\mathrm{x}} \sim 0.28 \mathrm{~nm}, \lambda$ is evaluated to be 2.2 and $1.7 \mu \mathrm{m}$ for 100 and $200 \mu \mathrm{N}$ indentation loads, respectively. These calculated values of $\lambda$ agree well with the value estimated from the mechanistic reasoning given earlier $(2.8 \mu \mathrm{m})$, and again are much larger than the plastic zone size derived from continuum prediction $(0.56 \mu \mathrm{m})$. The direction of dislocation glide relative to the line direction depends on various factors, such as grain size and grain orientation. Since the direction of dislocation glide is random, the distance over which dislocations approach the free surface varies more for a narrower line. In addition, the extension of equilibrium dislocation position rationalizes the increase of variation in indentation depth with decreasing line width.

\section{Conclusion}

The effect of length scale on the elasto-plastic mechanical response has been investigated via nanoindentation of patterned aluminum lines on a $\mathrm{Si}$ substrate. We find that narrower lines show a more compliant deformation during indentation. As the continuum based approach (both of FEM and analytical) could not rationalize this experimental trend, a model of discrete dislocation extension during the incipient stage of indentation has been proposed as a possible mechanism. Additionally, we find that there is no length scale effect on the elastic indentation response prior to the first plastic event. Although direct experimental evidence for such a mechanism has not been reported, the substantiation of the results in this study, may contribute significantly to our understanding the microplasticity at the incipient stage of nanoindentation, as well as the effects of length scale on the inelastic deformation of materials.

\section{Acknowledgements}

This work was supported by the Defense University Research Initiative on NanoTechnology (DURINT) on "Damage- and Failure-Resistant Nanostructured and Interfacial Materials" which is funded at the Massachusetts Institute of Technology (MIT) by the Office of Naval Research under grant N00014-01-1-0808.

\section{References}

[1] Nix WD. Metall Trans A 1989;20A:2217.

[2] Pharr GM, Oliver WC. MRS Bull 1992;7:28.

[3] Nix WD. Mater Sci Eng A 1997;234-236:37.

[4] Gouldstone A, Koh HJ, Zeng KY, Giannakopoulos AE, Suresh S. Acta Mater 2000;48:2277.

[5] Bahr DF, Kramer DE, Gerberich WW. Acta Mater 1998;46:3605.

[6] Suresh S, Neih TG, Choi BW. Scripta Mater 1999;41:951.

[7] Gerberich WW, Kramer DE, Tymiak NI, Volinsky AA, Bahr DF, Kriese MD. Acta Meter 1999;47:4115.

[8] Johnson KL. Contact mechanics. Cambridge, UK: Cambridge University Press; 1985.

[9] ABAQUS Version 6.1, Pawtucket, RI: Hibbitt, Karlsson \& Sorensen, Inc., 2000.

[10] McClintock FA, Argon AS, editors. Mechanical behavior of materials. Reading, MA: Addison-Wesley; 1966. p. 123. 